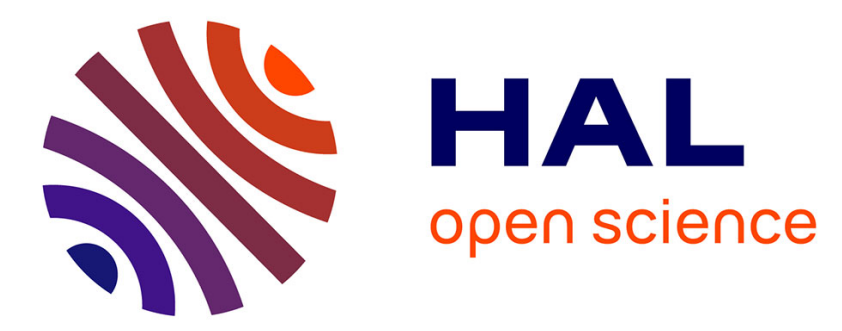

\title{
Réflexions sur une anthroponymie nobiliaire comparée entre la principauté de Morée et le royaume de Chypre, à travers la Chronique de Morée et les Lignages d'outremer
}

Isabelle Ortega

\section{To cite this version:}

Isabelle Ortega. Réflexions sur une anthroponymie nobiliaire comparée entre la principauté de Morée et le royaume de Chypre, à travers la Chronique de Morée et les Lignages d'outremer. Cahiers du Centre d'Etudes Chypriotes, 2013, 43 (1), pp.349-361. hal-03062897v2

\section{HAL Id: hal-03062897 \\ https://hal.science/hal-03062897v2}

Submitted on 15 Jun 2017

HAL is a multi-disciplinary open access archive for the deposit and dissemination of scientific research documents, whether they are published or not. The documents may come from teaching and research institutions in France or abroad, or from public or private research centers.
L'archive ouverte pluridisciplinaire HAL, est destinée au dépôt et à la diffusion de documents scientifiques de niveau recherche, publiés ou non, émanant des établissements d'enseignement et de recherche français ou étrangers, des laboratoires publics ou privés.

\section{(1) (1) $\$$}

Distributed under a Creative Commons Attribution - NonCommercial - NoDerivatives 44.0 


\title{
RÉFLEXIONS SUR UNE ANTHROPONYMIE NOBILIAIRE COMPARÉE ENTRE LA PRINCIPAUTÉ DE MORÉE ET LE ROYAUME DE CHYPRE, à travers la Chronique de Morée et les Lignages d'Outremer
}

Isabelle ORTEGA

\begin{abstract}
In the Middle Ages, the Principality of Morea and the Kingdom of Cypris are two comparable states in many areas, but studies comparing them are rare. The purpose of this anticle is to return to the anthroponymy of the two aristocratic societies in order to identify their common struchures and differences; beyond numerous similarities, each still retains its specifics.
\end{abstract}

\section{Introduction}

Que de points de comparaison entre la principauté de Morée et le royaume de Chypre! Deux bastions latins à l'est de la Méditerranée, deux communautés marquées par l'inftuence française dans de nombreux domaines et deux sociétés, bien entendu, dans lesquelles la noblesse, dont les membres revendiquent Jeur autorité par leurs origines familiales, leur richesse ou leur mérite, occupe une place impontante. Au sein de ce groupe social organisé en lignages, comprenant l'ensemble des individus descendant d'un ancêtre commun, conscients de leurs liens familiaux, les lignagers portent des marqueurs personnels, tels que les noms, sceaux, signatures ou encore blasons qui leur permettent d'affirmer à la fois leur singularité et leur appartenance à ce groupe.

Entre le $\mathrm{xI}^{\mathrm{e}}$ et le $\mathrm{XII}^{\mathrm{e}}$ siècle, en Occident, progressivement mais systématiquement, s'est opérée une évolution : les individus qui, jusque-là, étaient désignés par un seul nom portent désormais un surnom. Celui-ci est de nature différente selon le groupe social de son détenteur. Reflétant le plus souvent le métier exercé pour les plus modestes, il peut également reprendre le non du père voire d'un aïeul, ou un nom de lieu. Dans ce dernier cas de figure, il s'agit soit de la ville d'origine d'un individu qui s'est déplacé, soit pour les nobles du nom du château familial.

Le premier élément de dénomination, le nom de baptême, qui est devenu le prénom, est un marqueur d'identité, un facteur de transmission de biens symboliques, mais aussi fonciers, et la manière dont il circule dans la parenté reflète le degré de conscience familiale, tout en témoignant également des distinctions de sexes. Déjà en 1974, lors 
du colloque de Rome, Karl Ferdinand Werner précisait l'importance que devait revêtir l'étude approfondie des noms propres pour comprendre au mieux la politique dynastique des grandes maisons aristocratiques '. Depuis, un ensemble d'historiens, le groupe d'Azay-le-Ferron, a répondu à son appel en étudiant les sources dans de nombreuses régjons françaises et en regroupant leurs résultats anthroponymiques ${ }^{2}$. Il en a été de même pour le royaume de Jérusalem avec les travaux d'Iris Shagrid par exemple ${ }^{3}$. Leurs conclusions sont intéressantes et servent de fondement à d'autres réflexions, notamment la nôtue, or il n'est pas question dans cet article d'envisager tous les éléments constitutifs de la dénomination des individus, mais de livrer quelques réflexions générales quant à la prénomination en Morée et en Chypre, à des fins comparatives qui peuvent, nous semble$\mathrm{t}$-il , être éciairantes ${ }^{4}$.

Afin d'étudier at mieux l'anthroponynie, cette partie de l'onomastique qui étudie les noms de personnes, il fallait commencer notre recherche par des sources comparables. Notre choix s'est porté sur la Chronique de Morée. Ele est déclinée en plusieurs langues, mais nous allons surtout utiliser ici la version fançaise qui est une source majeure pour les années 1095-1292 ". Sans jamais renoncer à son parti pris, le chroniqueur dresse un tableau de la principauté en demi-teintes, partagé entre les récits des campagnes militaires et ceux de la vie quotidienne des grands seigneurs moréotes qui témoignent de leurs mentalités et de leurs conceptions familiales. Les spécialistes situent approximativement sa rédaction dans la première moitié du XJv" siècle ${ }^{6}$.

Les Lignages d'Outremer, quant à eux, étaient initialement insérés dans les Assises de Jérusalem, permettant ainsi aux juristes de disposer de références généalogiques pour compléter les livres de droit. Il existe plusieurs versions des Lignages $^{7}$, mais la plus ancienne peut être datée de 1265-1270 grâce à la précision des commentaires, tandis qu'une autre, remaniée, daterait de 1305-1306. Ces Lignages se présentent sous forme de chapitres portant comme titre le nom d'une dynastie, aussi bien du royaume de Chypre que des États de l'Orient latin et dans chacun de ces chapitres l'auteur, ou les auteurs plus vraisemblablement ${ }^{8}$, détaille/ent systématiquement les membres fondateurs puis les

1. Werner 1977, p. 31.

2. Ce nom est donné en référence aux rencontres annuelles qui se sont tentes à Azay-le-Ferron en 1986 et 1987, et qui ont donné lieu à la publication des cinq volumes sur la Genèse médiévale de l'anthroponymie moderne, sous la direction de Monique Bourin.

3. Shagrid 2003.

4. Si j'aj déjà pu étudier l'anthroponymie nobiliaise dans la principauté de Morée lors de mon doctorat (Ortega 2012), en ce qui concerne Chypre tout reste à faire pour moi et de nombreux indices sembient m'orienter vers des comparaisons possibles.

5. Longnon 1911.

6. Jacoby 1968, p. 135-136.

7. Nielen 2003, p. 25-32.

8. Nielen 2003 , p. $20-25$. 
mariages et la descendance. Cette source évoque de façon indifférenciée les dynasties du royaume de Chypre mais aussi celles des États latins d'Orient, car les mariages sont très nombreux entre ces deux sociêtés, rendant difficile l'étude de l'une sans l'autre.

À travers les sources précédemment énoncées, dans quelle mesure peut-on comparer l'anthroponymie nobiliaire moréote et chypriote? Pour cela, nous pourrons évoquer tout d'abord des sociétés nobiliaires ayant des modes de vie proches et une conscience de leur' groupe favorisant un système ononastique comparable, alors même que chaque groupe social conserve des caractéristiques propres.

\section{Des centres d'intérêt similaires}

Les historiens spécialistes d'anthroponymie s'entendent pour reconnaître que l'action même de nommer les individus est une affaire de famille, car la désignation sert aussì bien à marquer l'appartenance d'un individu à un groupe qu'à l'individualiser. Pour certains historiens, l'émergence du ménage, qui se démarque de l'ancienne structure lignagèrè occidentale, date du bas Moyen Âge ${ }^{9}$. Rien de tel en Motée, où le lignage, héritage des premiers conquérants, reste l'armature sociale de référence pour la noblesse qui, ainsi, affernit sa domination politique, économique et sociale sur le territoire. Le système f́éodal s'en est inspiré et tend à le suppléer lorsque les réseaux de parenté s'avèrent insuffisants ${ }^{\text {to. }}$.

Or l'influence occidentale dans le domaine de la parenté ne trouve pas un terrain vierge dans les Balkans, car les grandes familles grecques du Péloponnèse sont familiarisées avec ces structures, ce qui permet d'intégrer moins difficilement les lignages nobiliaires latins au sein de la principauté ${ }^{1}$. Cette organisation familiale se retrouve en Chypre où la société peut être perçue comme une sorte de laboratoire oì se confrontent culıture autochtone et culture allochtone ${ }^{\mathrm{i} 2}$. La noblesse chypriote, connaît elle aussi la structure de parenté lignagère, it n'est qu'à voir le titre même de la source que nous prenons ici comme référence: " $|\ldots|$ Les Lignages sont une source irremplaçable qui nous livre le tableau d'une classe sociale telle qu'elle se voyait elle-même, ce qui ne va pas sans des reconsinictions, des oublis $|\ldots|{ }^{13}$. $\AA$ reprendre les mots de Jean Richard, on retient

9. Cette hypothèse est tout de même remise en cause depuis plusieurs années par les historiens de la famille. La documentation témoigne des vives résistances de la parenté face à l'émergence de l'individı : ce dernier reste vulnérable dans une société marquée par la solidarité. Le passage de la fartilte large du haut Moyen Âge à la famille nucléaire de l'époque modeme n'a pas livré touș ses mystères (Fossier 1986, p. 156 ; Bresc 1986, p. 170).

10. Burguière 1990, p. 390 .

11. Le vocabulaire de la parenté à Byzance révèle la présence de structures horizontales et verticales comparables à celles de l'Occident. Concernant les lignages plus précisément, l'indifférenciation des lignées est un héritage romain tardif dont la tendance s'accrôt au fil des siècles (Patlagean 1986, p. 219).

12. Fourrier, Grivaud 2006, p. 407.

13. Avant-propos de Jean Richard (Nielen 2003, p. 8). 
que l'un des aspects les plus intéressants pour les historiens est d'étudier l'image qu'un groupe social peut avoir de lui-même et les Lignages d'Outremer témoignent du souvenir que les nobles chypriotes veulent laisser à la postérité. Une noblesse que certains jugeront "femée », puisque de nombreuses dispenses matrimoniales sont demandées et obtenues, pour autoriser des mariages à degrés de parenté rapprochés ${ }^{14}$, et que d'autres qualifieront d' « ouverte », car de multiples mariages sont organisés avec les États latins de SyriePalestine, voire avec le royaume arménien voisin ${ }^{15}$.

Non seulement les noblesses de Morée et de Chypre sont organisées d'une façon comparable, mais en outre, à plusjeurs reprises dans leurs histoires respectives, ces deux États nouent des relations politiques et militaires, comme lors du séjour de Louis IX en Chypre. Au printemps 1249, le prince de Morée, Guillaume II de Villehardouin, s'embarque avec vingt-quatre vaisseaux et quatre cents hommes pour participer à la croisade du roi de France ${ }^{16}$ qu'il rejoint en Chypre ${ }^{17}$. Ces chevaliers semblent avoir fait impression sur l'entourage du roi car leur participation à la croisade est souhaitée ${ }^{18}$. La principauté rếunit ainsi plusieurs avantages aux yeux de Jean de Joinville, proche du souverain français : elle est réputée pour être une terre de chevalerie et se trouve à peu de distance des États latins d'Orient. Si ce conseil est formulé, c'est en raison de la réputation des chevaliers de Morée et du souvenir laissé par Guillaume II lors de la croisade. En participant à celle-ci, les chevaliers remplissent l'iłéal du miles Christi allant combattre les infidèles pour la défense de leur religion ; c'est également l'occasion pour toutes les troupes chrétiennes de se rencontrer, de se découvrir et de $s$ 'estimer.

Dans les deux sources, il est intéressant de noter les mêmes conceptions lignagères : les parents nombreux sur lesquels on peut compter, le prestige d'un ascendant de poids, fondateur de cette dynastie, ou encore l'importance des filles que l'on va marier. Autant d'intérêts communs qui attestent d'une même conception de la famille et des moyens mis en place pour les défendre. Pour reprendre ces différents thèmes, on peut noter effectivement le poids des lignagers aussi bien dans une source que dans l'autre. Les fratries sont bien détaillées dans l'ouvrage de généalogie que constituent les Lignages d'Outremer ${ }^{19}$, alors que seuls les personnages d'envergure apparaissent dans une source narrative telle que la chronique ${ }^{20}$. Les deux sources font tout de même une place au

14. Rudt de Collenberg 1977.

15. Nielen 2003, p. 131-140.

16. Richard, 1976, p. 229-231.

17. Joinville $1995, \S 148$.

18. Joinville 1995 , § 427 : « Si mette le roy ses deniers en despense, et envoit le roy querre chevaliers en la Moree et outre mer. Et quand l'en orra nouvelles li venront de toutes pars, par quoy il pourra tenir heberges un an, se Dieu plet [...]».

19. Chaque chapitre détaille génération après génération les fratries, leurs alliances et leurs descendances (Nielen 2003, p. 60-62, à titre d'exemple).

20. Pour attant on peut noter la place accordée aux cadets et aux collatéraux (Ortega 2012, p. 175-179). 
fondateur de la dynastie : il apparaît en tête de chapitre dans la source chypriote, on lui accorde une place parmi les premiers conquérants dans la chronique ${ }^{21}$ : responsable d'une segmentation de lignage, il devient le fondateur de la dynastie outre-mer ${ }^{22}$. Quant aux filles que le lignage va marier, elles sont tout aussi importantes dans une source que dans l'autre : leurs mariages sont systématiquement mentionnés dans les Lignages d'Outremer et ils sont également considérés comme primordiaux dans la Chronique de Morée, bien que, le plus souvent, le chroniqueur ne mentionne pas le nom de l'épouse en question ${ }^{23}$.

Au-delà de ces points communs, assez généraux, il y a quelques cas précis d'alliances matrimoniales dans ces sources. À l'instar de Hugues de Brienne, qui épousa Isabelle de La Roche,

«Hugue fu cuens de Brene et espousa Ysabeau, la flle dou duc d'Athanes, qui avoit esté feme dou seignor de Karitaine et orent .l. fis et whe fille, Gantier et Agnès " ${ }^{24}$.

"Mais ne denora mie longuement que li dux Guis de la Roche. quifreres estoit de celle dame, par la voulenté et consentement dou roy et dou prince, si ordina et fist le mariage de celle dame sa stuer et du noble homme messire Higues, le conte de Brene et de Liche $|\ldots|{ }^{25}$.

Sa mère étant une Lusignan, Hugues appartient à une grande famille de l'Orient latin, et à ce titre il est mentionné dans les Lignages d'Outremer. Passé au service de Charles d'Anjou, il intervient dans la principauté de Morée après les traités de Viterbe de 1267 , et il épouse alors Isabelle de La Roche, de la dynastie régnant sur le duché d'Athènes. Elle est alors veuve d'un baron moréote ${ }^{26}$, et il est intéressant de voir que, de façon indirecte, cette source évoque Geoffroy de Briel, baron de Karytaina. Neveu du prince Guillaume de Villehardouin, il est mis en avant à plusieurs reprises dans la Chronique de Morée, car c'est un farouche défenseur de la principauté face aux Grecs. Sa vie romanesque, ses frasques et ses actes impétueux ont séduit les chroniqueurs ${ }^{27}$, lui assurant une renommée au-delà des mers.

Une autre alliance témoigne du rapprochement entre les deux noblesses : c'est celle qui unit Nicolas II de Saint-Omer, co-seigneur de Thèbes et baile de la principauté dans la deuxième moitié du XII] siècle, à Marie d'Antioche, scour du prince d'Antioche :

«Bohémond fut le septième prince et épousa Marguterite $\lceil$... J. Sa saur Isabelle mourut anssi, et l'autre soutr, Marie, devint la fenme de Nicolas de Saint-Omer ${ }^{28}$.

21. Peu après la conquête, les principaux conquuếtants sont cités (Longnon 1911, § 128).

22. Ortega 2012 , p. 109.

23. Ortega 2012, p. 556-561.

24. Nielen 2003 , p. 90.

25. Longnon 1911, \$498.

26. Ortega 2012, p. 583, 620 .

27. Ortega 2001, p. 329-341.

28. Nielen 2003, p. 145. 
«Cellhi monseignor Nicole $|\ldots|$ fit riches desmesurement dou grant avoir que il prist a la princesse d'Antioche ${ }^{20}$.

C'est un mariage qui reste atypique dans le paysage nobiliaire moréote, et les chroniqueurs n'ont de cesse de rappeler que cette union fit la fortune des Saint-Omer ${ }^{30}$, seigneurs qui sont par ailleurs, ou par conséquence, très puissants au toumant des $x I^{*}{ }^{*}$ Xгv" siècles.

Toutefois, ces exemples restent relativement isolés, car on pourrait s'attendre à davantage d'unions entre ces sociétés aux multiples points communs, à l'image des nombreux liens de parenté tissés entre le royaume de Chypre et les États latins d'Orient. Peut-être la Morée apparaît-elle excentrée alors, relevant de l'Empire latin de Constantinople jusqu'en 1261, puis des Angevius de Naples ensuite ${ }^{31}$. Pourtant des ressembiances peuvent être notées entre la principauté et le royaume, notamment dans les prénominations employées au sein des familles nobles.

\section{Un système onomastique comparable}

Le système binominal a cours dans ces États latins de Méditerranée, où les nobles latins importent le système de dénomination utjlisế dans leurs provinces d'origine depuis les $\mathrm{XI}^{\mathrm{s}}$-XII ${ }^{\mathrm{e}}$ siècles ${ }^{32}$. Il comporte deux éléments nettement distincts : le prénon qui se rapporte à l'individu, tandis que le second est soit un nom de famille quì incarne le lignage, soit un toponyme qui représente le patrimoine. Étant donné que le système familial offre des sinilitudes dans ces sociétés, il constitue le terreau favorable pour le développement d'une organisation anthroponymique comparable.

Assurément, l'infiuence onomastique occidentale se fait sentir en Morée où les prénoms dominants sont analogues à ceux qui sont les plus dévolus durant la même période en Occident. Il est remarquable de noter que la concentration des prénoms masculins y est plus grande que pour les femmes, beaucoup plus d'hommes portant un même prénom que les femmes ${ }^{33}$. En effet, 1 'importance attribuée au nomem proprium en fait un élément stratégique pour les héritiers, mais en ce qui concerne les filles, qui dans tous les cas vont sortir du lignage lors de leur mariage, il n'y a pas la même détermination.

Au $\mathrm{Xul}^{\mathrm{e}}$ siècle dans le royaume de France, la forme la plus courante de désignation féminine pour les scribes médiévaux est un prénom suivi d'une place familiale ${ }^{34}$. Cela permet de noter la situation des femmes au sein des lignages d'origine et par alliance ; en outre c'est un moyen d'éviter l'homonymie. Les études menées semblent converger sur un point : si le stock onomastique féminin paraît plus riche que le stock masculin, il suit

29. Longnon $1911, \S 554$.

30. Ortega 2012 , p. $607-608,644$; Longnon $1911, \S 553$.

31. Ortega 2012, p. 14-17.

32. Bourin 1987, t. I, p. 7-14.

33. Ortega 2012 , p. 457-458.

34. Bourin 1992, p. 2. 
cependant la même réduction observable aux XI" et XII" siècles. Les règles de dévolution sont moins contraignantes pour les filles et l'évolution se fait en faveur des noms tirés de noms masculins et des noms de saintes ${ }^{35}$. Finalement dans te royaume de France, se distingue une aire septentrionale avec des prénoms dominants comme Marguerite, Isabelle et ses dérivés (Alix, Élisabeth), Agnès et Jeanne ${ }^{36}$; et pour les hommes les prénoms les plus répandus sont Jean, Guillaume, Pierre et Robert ${ }^{37}$.

Les prédispositions par origine sont intéressantes car les lignages n'affectionnent pas les mêmes prénoms. Dans la principauté de Morée, ils reproduisent les tendances qui se dessinaient dans leurs régions d'origine au xuI" siècle. Les Italiens, très présents dans l'Archipel notamment, affectionnent particulièrement les noms religieux comme Nicolas, Jacques ou François, le nouveau saint mendiant ${ }^{38}$. Il faut reconnaître que l'Église a poussé les fidèles à choisir les noms de grands saints évangéliques ou fondateurs d'ordres religieux et le baptisé établit de la sorte un lien avec le divin, qui lui assure en contrepartie une protection. Les lignagers d'origine française apprécient certes les noms de tradition J'eligieuse tels que Jean, mais l'inclination est moins marquée que pour les Italiens, car ils entretiennent leur penchant pour les prénoms germaniques. Ces derniers sont également ceux dévolus aux princes de la maison Villehardouin comme Geoffroy, Guillaume ${ }^{39}$; leur présence, de même que celle d'Isabelle, parmi les désignations les plus attribuées n'est pas un hasard car ces souverains ont été appréciés, d'ailleurs leurs prénoms correspondent à une vogue dans les régions du nord-est français ${ }^{4}$. 11 n'est pas rare de donner le nom du prince à un cadet dans un désir de protection spirituelle en vue du salut ou dans une volonté d'afficher sa vassalité : la cause réelle est difficile à déterminer ${ }^{41}$. La concentration des choix sur les prénoms princiers est expliquée en partie par l'attente de protection de la part du prince tutélaire ${ }^{42}$ el l'on retrouve cette volonté auprès des vilains qui affectionnent particulièrement le prénom Guillelmus ${ }^{43}$.

Les Lignages d'Outremer, quant à eux, Jivrent un échantillonnage des prénoms en vogue dans la noblesse chypriote. Ce texte mentionne environ mille personnes sur deux siècles environ, et parmi les prénoms cités certains reviennent souvent: Jean, Hugues,

35. Guyotjeannin 1996, p. 386.

36. Bourin, Chareigne 1992, p. 227.

37. Bourlet 1992, p. 13-14; Saluvage 1992, p. 55.

38. Saint-Guillain 2003, p. 10-12 ; Ortega 2012, p. 554.

39. Ortega 2012, p.569-571, 613 ; Longnon 1911, § 108, 109, 195, 198, entre autres.

40. On retrouve parmi les prénoms féminins les plus à la mode en Morée, les mêmes que dans le nord du royaume de France (Ortega 2012, p. 456 459).

41. Bourin 1996, p. 190.

42. Bourin [996, p. 195.

43. Une étude des dénominations des plus modestes a déjà èté faite (Longnon, Topping 1969, p. 221). 
Guillaume, Guy ou Philippe, pour les femmes Isabelle, Marguerite, Marie, Alix, Agnès ${ }^{44}$. Autant de prénoms comparables à la Morée et à l'Occident, car on y retrouve l'influence des saints apostoliques, l'importance des personnages bibliques et des prénoms germaniques également ${ }^{45}$. De plus, les prénoms portés par la dynastie régnante des Lusignan suivent la mode ou la précèdent, tant et si bien que l'on ne peut que noter la corrélation entre les choix princiers et ceux de la population.

Il serait intéressant d'étudier le système de dévolution des prénoms dans les deux contrées, pour autant ce sera l'objet d'un prochain travail, car il faudrait pour cela compléter notre approche par bien d'autres sources. Autant de renseignements qui permettraient de recréer des généalogies, indispensables pour étudier les dévolutions. Toutefois quelques réflexions peuvent être menées dans ce domaine, car plusieurs générations peuvent apparaître dans la Chronique de Morée, que l'on peut comparer ainsi à la source éminemment généalogique que constituent les Lignages d'Outremer. On peut citer les Autremencourt, seigneurs de Salona, qui sont de grands barons moréotes et qui utilisent un prénom récurent, Thomas, directement lié au patrimoine ${ }^{46}$. C'est un prénom peu répandu au xul" siècle en Morée et qui permet de les reconnaître aisément dans les actes ${ }^{47}$, car les seigneurs de Salona se le transmettent en ligne directe. Le rapport semble dès lors evident entre l'anthroponymie et les pratiques successorales : par le choix de la dénomination, le lien de filiation est explicité et ainsi des droits peuvent être revendiqués.

Mais au-delà des biens matériels, l'anthroponymie intègre la transmission dans un sens plus large pouvant comprendre un rôle politique, une situation de puissance ou encore une réputation sociale. Le seigneur țansmet à son fils ses domaines patrimoniaux, ses offices, son titre et son nom : le tout permet à l'héritier d'accéder aux pouvoirs du père. C'est pour cela que les dénominations ne sont jamais le fruit du hasard et révèlent les stratégies des ligrages.

On note la même récurrence de quelques prénoms, propres à certaines familles, dans les Lignages d'Outremer. Ces prénoms, emblématiques d'une dynastie comme Balian chez les Ibelin ${ }^{48}$, représentent une tendance que l'on retrouve aussi en Occident dans les plus grandes familles où l'on distingue ainsi les héritiers. La dynastie des Autremencourt adopte ce système mais la transmission collatérale continue également à être utilisée ${ }^{49}$. Dans la famille princière par exemple, le prénom Geoffroy est porté par deux membres de la famille, car c'est un symbole dynastique fort représentant le fondateur du lignage des Villehardouin en Morée. Geoffroy I ${ }^{\mathrm{er}}$ est à l'origine de la segmentation du lignage champenois, devenant le représentant des conquérants fiancs de cette principauté.

44. Nielen 2003, p. 28.

45. Shagrid 2003, p. 23-36.

46. Longnon $1911, \$ 238 ;$ Ottega 2012, p. $579-581$ et 618 .

47. Longnon 1937 , p. 19-20.

48. Nielen 2003, p. 61-63.

49. Ortega 2012, p. $461-463$. 
Une autre similitude entre la Chronique de Morée et les Lignages d'Outremer apparaît pour les prénominations. Ce sont les hypocoristiques, ces diminutifs affectueux que les femmes portent facilement dans les grandes familles ${ }^{50}$ : Marion, Jeharette, Gilète, Guillemette sont autant de déformations de prénoms classiques ${ }^{51}$ qu'il ne faut pas sousestimer, car ces formes dérivées caractérisent une situation historique ${ }^{52}$ qui témoigne de la représentation que le lignage veut donner de lui-même. Dans la principauté de Morée, nous retrouvons les mêmes tendances qu'en Occident, c'est-à-dire des diminutifs affectueux qui ne se transmettent pas et qui restent des marqueurs individuels, notamment Jeannette, pour Jeanne de Brienne, Guyot pour qualifier Guy If de la Roche, mais ils sont relativement rares ${ }^{53}$. Inversement, dans les Lignages d'Outremer il y a davantage d'exemples, alors que le texte est beaucoup plus court. On note ainsi la mention d'un Peretin, Thomassin, Guiotin, Guillemin ${ }^{54}$. Autant dire qu'il s'agit de la reprise de prénoms très courants, auxquels on rajoute un suffixe, le plus souvent pour différencier le fils du père, voire du grand-père. Mais il est possible, bien que plus rare, de vouloir différencier la fille de la mère : c'est le cas de Sebilon, dont la mère est Sebile le Petit ${ }^{55}$. Ainsi, ces nobles chypriotes ou moréotes font des choix comparables lorsqu'il s'agit de prénommer leurs enfants au moment des baptêmes, cependant ils conservent également des traits propres.

\section{Des singularités conservécs}

La famille choisit le nom de l'enfant, soit à la naissance, soit pour le baptême et plusieurs possibilités s'offrent à elle. L'usage de certains prénoms a déjà été sanctionné. par les ancêtres et, génération après génération, ils symbolisent l'affirmation de la dynastie ; toutefois, l'attrait de la nouveauté peut charmer certaines familles, notamment en ce qui concerne les dévolutions féminines. Il faut noter cependant la difficulté qui entoure une étude de la césignation féminine, car les femmes dont la présence dans les actes est très discrète ne sont guère plus représentées dans les sources narratives : toute réflexion les concernant se construit donc sur des effectifs très faibles. Ainsi, alors que la dênomination masculine est déjà peu précise, il est encore plus difficile de cenner l'onomastique féminine, souvent omise par les sources. Dans le royaume de France, les femmes sont le plus souvent désignées comme épouses, mères, filles, sours d'un tel : autant d'expressions qui font écran à la connaissance d'un éventuel nom, voire d'un

50. Bourin 1992, p. 3.

51. Sauvage 1992, p. 61.

52. Klapish-Zuber 1984 , p. 38 .

53. Longnon $1911, \$ 500,550 ;$ Ortega 2012, p. $471-472$.

54. Nielen 2003 , p. $64,65,69,72,118-119$.

55. Nielen 2003 , p. 128. 
surnom ${ }^{56}$. C'est exactement le cas de ia Chronique de Morée marquée par les confusions qui jalonnent le récit, Jes incertitudes orthographiques qui le ponctuent, parfois même les prénoms qui sont confondus ou omis. Néanmoins, l'intérêt est de dégager des tendances ou une évolution dans les pratiques.

Proportionnellement, une majorité écrasante de femmes ne sont même pas dénommées. Dix femmes seulement sont prénommées dans la Chronique de Morée sur 1024 paragraphes ${ }^{77}$ ! Le chroniqueur connaît leur existence, elles apparaissent comme les maillons de la chaine généalogique, comme l'enjeu des tractations politiques ou matrimoniales, mais leur identification précise importe peu. Ce qui compte, avant tout, c'est de pouvoir les situer dans leur lignage ou vis-à-vis de leurs alliés. L'épouse reste finalement dans l'ombre du père, de l'époux ou du fils héritier, et ainsi sont perdus de précieux renseignements pour l'anthroponymie féminine. Si cette tendance se retrouve également en Occident ${ }^{58}$, cela rend la comparaison avec Chypre fragile, car inversement, dans les Lignages d'Outremer, l'anthroponymie féminine n'est pas occultée. En effet, dans chaque chapitre de cette source, les épouses, mères, filles, sours apparaissent nommément ${ }^{59}$.

Autre nuance, dans les Lignages d'Outremer une plus grande liberté semble marquer les désignations féminines. En effet, on trouve des Tourterelle, Plaisance, Douce on Bienvenue, autant de prénoms auguratifs que l'on peut retrouver également en Occident ${ }^{60}$, tandis que le chroniqueur moréote, lui, n'atteste pas autant de liberté dans les choix de prénoms, fussent-ils féminins. En dehors de cette source narrative, on sait par ailleurs qu'il y a quelques prénoms auguratifs attestés, mais ils sont bien moins nombreux qu'en Chypre ${ }^{61}$.

Les formes de dénominations privilégiées dans la Chronique de Morée sont donc : prénom + nom + fonction, ou prénom + toponyme, comme en Occident. Il s'agit d'une permanence vis-à-vis des provinces d'origine des nobles moréotes, où l'on retroltve les mêmes caractéristiques anthroponyniques. Au début du Xive siècle, date de rédaction de cette source narrative, les nobles se dénomment majoritairement à l'occidentale, tandis que l'influence grecque se fait sentir dans les surnominations. $C^{\prime}$ est surtout dans le duché de l'Archipel que les dynasties usent de cognomina dont certains ont une sonorité grecque. À l'instar de Giovanni II Ghisi, dit Zannaki, dont le sumom en aki provient

\section{Barthelemy 1992, p. 73.}

57. Ortega 2012 , p. 556-561.

58. Bourin 1992, p. $1-9$.

59. Tous les clapitres sont sur le même modèle (Nielen 2003).

60. Nielen 2003, p. $65,67,72,114$, entre autres. On note même la présence d'une Bourgogne, ant prénom si atypique qu'il ne peut que rappeler l'attachement que ces familles d'outre-mer peuvent avoir vis-à-vis de leurs provinces d'origine (ibid., p. 61).

61. A l'image de Bonne de la Roche, appartenant à la famille des seigneurs d'Athènes d'origine bourguignonne. C'est d'ailleurs un prénom qui est attesté dans cette province (Beck 1992, p. 98 ; Ortega 2012 , p. 599-600). 
d'un suffixe grec qui renvoie à l'attachement et permet de le distinguer de son grandpère paternel ${ }^{62}$. D'autres diminutifs d'inspiration indigène apparaissent dans les sources, comme Giovanni Ir Sanudo appelé Januli, ou Francesco Crispo dit Franguli ${ }^{63}$. Dans tous les cas, ils ne se transmettent pas et ils restent des marqueurs individuels.

Le système de dénomination des Latins mentionné dans la Chronique de Morée est donc conforme à celui développé en Occident aux mêmes siècles. Les dénominations masculines sont mieux connues que celles des femmes, et surtout plus complètes, associant le nom de famille, le toponyme et la fonction voire le cognomen. Les prénominations féminines, quant à elles, sont le plus souvent passées sous silence, mais certaines parviennent toutefois à se glisser dans la rédaction grâce aux références faites à leurs parents ou à leurs alliés. Elles ne sont donc pas tout à fait oubliées, surtout si elles représentent un important héritage ou une union matrimoniale profitable. Il y a en outre plusieurs mentions d'inspiration grecque, dont on ne trouve pas l'équivalent dans les Lignages d'Outremer. Cette source nous livre néanmoins un témoignage beaucoup plus complet et détaillé en ce qui concerne l'anthroponymie, dans lequel les femmes occupent une place de choix aux côtés des hommes de la dynastie.

\section{Conclusion}

L'importance accordée à l'attribution des prénoms reflète donc la dynamique lignagère et éclaire a posteriori l'histoire de la filiation familiale. La naissance, les atmes et le nom font partie d'une conscience lignagère. Ainsi ces structures de parenté voient-elles circuler entre elles des biens matériels ou symboliques (prénoms et honneur), des richesses et des individus lors des unions matrimoniales. C'est pour cela que les dénominations, au même titre que les biens fonciers, sont un atout dans le jeu social, car des règles de transmission assez strictes interviennent dans le choix des prénoms, analogues aux règles qui président à la circulation des patrimoines. Le nom se prête d'ailleurs à une approche pluridisciplinaire et, si le simple relevé de prénom est insuffisant en lui-même, il importe de l'analyser à la lueur du rang de naissance, de la puissance de la parentèle ou du destin des aînés : tous ces éléments éclairent des choix qui ne doivent rien au hasard. En effet, étant donné que les prénoms dynastiques renvoient l'image valorisante du groupe, il est indispensable de bien les choisir. Au même titre que la parenté suggérée par le patrimoine, l'usage d'un nom d'ancêtre explicite lui aussi les Iiens familiaux.

Cette modeste étude comparative de l'anthroponymie en Morée et en Chypre porte la promesse de pistes de recherche concernant les nobles latins. Le resserrement des stocks semble y être général comme en Occident mais à des rythmes différents, ainsi les conclusions résultant de cette étude onomastique partielle sont-elles conformes aux remarques faites par le groupe d'Azay-le-Ferron. L'intérêt est de poursuivre cette recherche en compulsant les sources ef en reconstituant les généalogies des lignages

62. Ortega 2012, p. $470-473$ et 636.

63. Slot 1982, p. 64. 
nobiliaires que l'on pourra ainsi aisément comparer ; mais on semble d'ores et déjà aboutir à une vaste Europe chrétienne des noms, incorporant la Morée, le royaume de Chypre et les États latins d'Orient qui usent de prénoms similaires à ceux employés dans les provinces d'origine des nobles moréotes.

Université de Nônes

\section{BIBLIOGRAPHIE}

BARTHÉlÉMY (D), 1992, «Éléments d'anthroponymie féminine d'après le cartulaire du Ronceray d'Angers (1028-1184) », dans Bourin 1987-2002, t. II, p. 67-80.

BECK (P), 1992, « Anthroponymie et désignation des femmes en Bourgogne au Moyen Âge $\left(\mathrm{X}^{\mathrm{e}}\right.$-XIV" siècle) $»$, dans Bourin 1987-2002, t. II, p. $89-100$.

Bounin (M.) ed., 1987-2002, Genèse médiénale de l'anthroponymie moderne, Tours, 5 vol.

Bourzin (M.), 1992, « Les difficultés d'une élude de la désignation des femmes ", dans Bourin 1987-2002, t, II, p. I-8.

Bourin (M.), Chareigne (P.), 1992, « Insignis femina, virilis femina ", dans Bourin 19872002 , t. 1I, p. 207-230.

Boturin (M.), 1996, « France del Midi el France du Nord : deux systèmes anthroponymiques ? ", dans M. Bouris, J.-M. Martin, Fr, Menant (éd.), L'Anthroponymie document de l'histoire sociale des mondes méditerranéens médiévaur. Actes du collogue international organisé par l'École Française de Rome (6-8 oct. 1994), Rome, p. 179-202.

Boukl.Er (C.), 1992, « L'antłroponymie à Paris à la fin du Xl1 ${ }^{2}$ siècle d'après les rôles de la taille du règne de Plilippe le Bel $»$, dans Bouriu 1987-2002, t. II, p. 9-44.

Bresc (H.), 1986, "L'Europe des villes et des campagnes ( $\mathrm{xul}^{\mathrm{L}} \mathrm{-} \mathrm{XV}^{\mathrm{c}}$ siècle) ", dans A. Burguière (dir.), Histoire de la famille, Paris, t. 1l, p. 169-211.

BuRGulère (A.), 1990, « Les "liens du sang". Marc Bloch, historien de la parenté $»$, dans H. Atsma, A. Burguière (éd.), Marc Bloch aujourd'hui. Histoine comparée et sciences sociales, Paris, p. 389-402.
Fossier (R.), 1986, " L'ère féodale ( $\mathrm{XI}^{\mathrm{F}} \mathrm{XII \textrm {X } ^ { \mathrm { c } }}$ siècle) », dans A. Burguière (dir.), Histoire de la famille, Paris, t. II, p. 141-168.

Fourkier (S.). Grivaud (G.), 2006. "L'écheveau », dans S. Fourrier, G. Grivaud (dir.), Identitếs croisées en un milieu méditerranéen : le cas de Chypre (AntiquitéMoyen Age), Mont-Saint-Aignan, p. 407-430.

GuYotJEAnNIN (O.), 1996, "Les filles, les femmes, le lignage ", dans M. Bourin, J.M. Martin, Fr. Menant (éd.), L'Anthroporymie documem de l'histoire sociale des mondes méditerranéens médiévaux, Rome, 1996, p. $383-400$.

JACOBY (D.), 1968, « Quelques considérations sur la "Chronique de Morée" ", Journal des Savants, p. 135-136.

Jorvyllite (J. de), 1995, La Vie de saint Louis, éd. J. Monfrin, Paris.

KLAPISH-Zuber (Chr), 1984, « Constitution et variations temporelles des stocks de prénoms $\%$, dans J. Dupâquier, A. Bideau, M.-É. Ducreux (éd.), Le Prénom. Mode et Histoire. Entretiens de Malher 1980, Paris, p. $37-48$.

LONGNON (J.) éd., 1911, Le Livre de la conqueste de la princée de l'Anorée. Chronique de Morée (I204-I305), Paris.

LoNGINON (J.), 1937, « Les Autremencout, seigneurs de Salona en Grèce (1204-1311) ", Bulletin de la Société Historique de HautePicardie 15. p. 15 48.

LoNGNON (J.), Topping (P.), 1969, Documents sur le régime des terres dans la principauté de Morée an XIN siècle, Paris et La Haye.

Nisulfin (M.-A.) éd., 2003, Lignages d'Ottremer, Paris. 
Ortega (1.), 2001, « Geoffroy de Briel, un chevalier au grand cour », Byzantinistica 3 , p. 329-341,

OrTEGA (I.), 2012, Les Lignages nobiliaires dans la Morée latine ( $\mathrm{x}\left(\mathrm{r}^{\mathrm{i}}-\mathrm{XV}\right.$ siècles). Permanences et mutations, Turnhout.

PATLAGEAN (É.), 1986, « Familles et parentèles à Byzance ", dans A. Burguière (dir.), Histoire de la famille, Paris, t. I1, p. 213-240.

RICHARD (J.), 1976, « Saint Louis dans l'histoire des croisades ", Bulletin de la Société d'émulation du Bourbonnais 55, p. 229-231 [repr. dans J. Richard. Orient et Occident at Moyen Age : contacts et relations (XIF-Xwe siècles), Londres, 1976 , étude $\mathrm{n}^{\circ} \mathrm{V}$ l.

Rudt de Collenbers (W. H.), 1977, « Les dispenses matrimoniales accordées à l'Orient latin selon les registres du Vatican d'Honorius III à Clément VII (1223-1385) ». Mélanges de ['École française de Rome 89, p. 1]-93.

RuIT de COLleniberg (W.H.), 1979-1980,

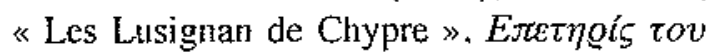

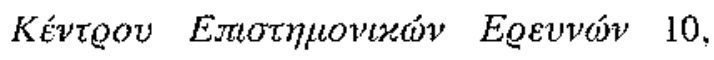
p. 85-319.

SAINT-GuLitaIN (G.), 2003, L'Archipel des seigneurs. Pouvoirs, société et insularités dans les Cyclades a l'époque de la domination latine $\left(\mathrm{X} / \mathrm{F}^{\mathrm{f}} \mathrm{X} \mathrm{V}^{\mathrm{F}}\right.$ siècles$)$, thèse de doctorat, Universite Paris I, 4 vol.

Sauvage (J.-P), 1992, \& Formes anthroponymiques féminines à Blois à travers une liste d'habitants de $1334 \%$, dans Bourin 1987 2002 , t. 1l, p. 45-66.

SHAGRID (I.), 2003, Naming Patterns in the Latin Kingdom of Jerusalem, $\mathrm{Oxford}$.

SLOF (B.J.), 1982, Archipelagus Thrbatts. Les Cyclades entre colonisation latine et occupation ottomane (1500-1718), Istanbul, 2 vol.

WERNER (K.F.), 1977, « Liens de parenté et noms de personnes », dans G. Duby, J. Le Goff (éd.), Famille et parenté dans l'Occident médiéval, Actes du colloque de Paris (6-8 juin 1974), Rone, p. 25-34. 